\title{
PHYTOCHEMICAL AND BIOLOGICAL INVESTIGA- TIONS OF FLACOURTIA CATAPHRACTA ROXB. CULTIVATED IN EGYPT
}

Hanaa M. Sayed ${ }^{1}$, Mahmoud H. Mohamed ${ }^{2}$, Faten M. Darwish ${ }^{1}$ and Ahmed M. Mohamed ${ }^{2}$

${ }^{1}$ Department of Pharmacognosy, Faculty of Pharmacy, Assiut University, Assiut, Egypt

${ }^{2}$ Department of Pharmacognosy, Faculty of Pharmacy, Al-Azhar University, Assiut, Egypt

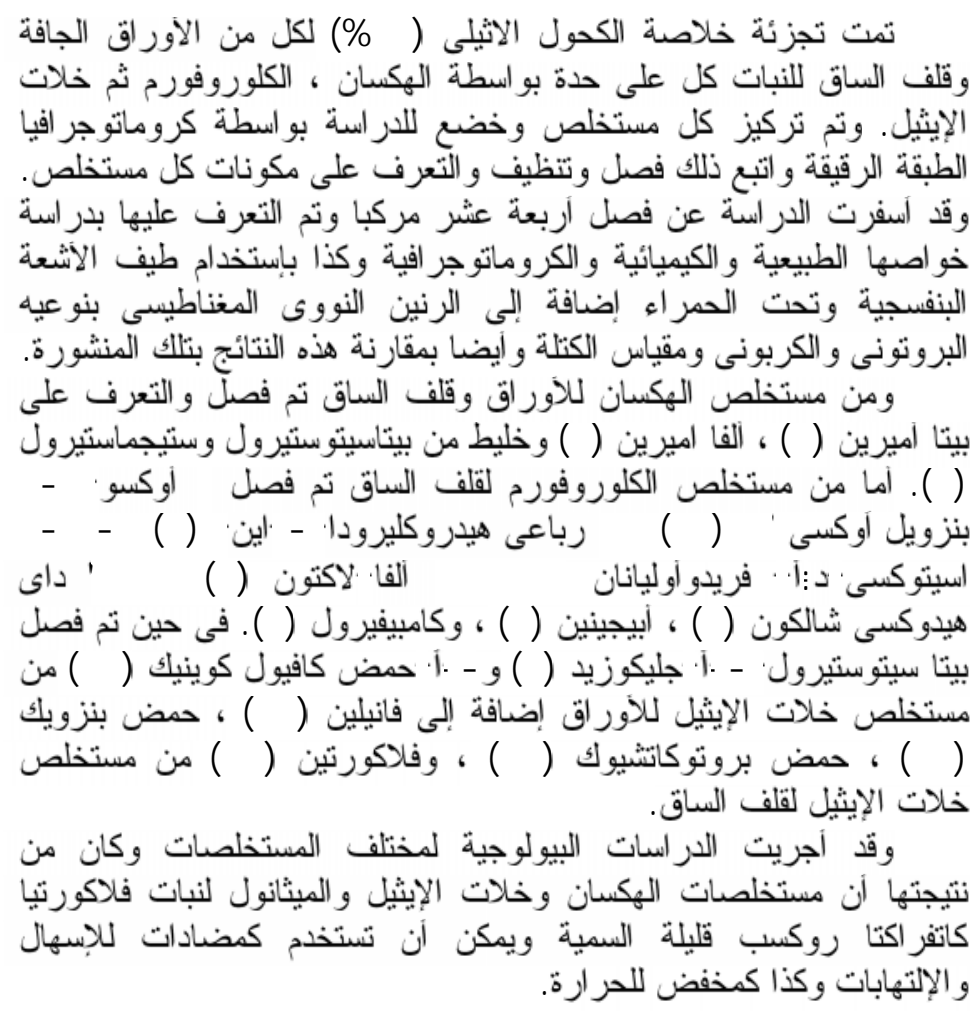

The concentrated $70 \%$ ethanolic extracts of the air-dried powdered leaves and stem bark of Flacourtia cataphracta were subjected separately to solvent fractionation by partitioning using

Received in 31/7/2006 \& Accepted in 30/10/2006 
n-hexane, chloroform and ethyl acetate respectively. Each concentrated fraction was subjected to TLC followed by isolation, purification and identification of the available constituents.

Fourteen compounds were isolated and identified by different spectral tools (UV, IR, ${ }^{1} H$-NMR, $\left.{ }^{13} C-N M R, M S\right)$ and comparison with corresponding literature data. $\beta$-amyrin (1), $\alpha$-amyrin (2), and a mixture of $\beta$-sitosterol and stigmasterol (3) were isolated from $n$ hexane fraction of both leaves and stem bark. 2-Oxo-18benzolyloxy-13(16), 14-tetrahydrocleroda-3-ene (4), 3- $\beta$-acetoxyD:A friedo oleanan-27,16 $\alpha$-lactone (5), 4,4'-dihydroxychalcone (6), apigenin (7) and kampferol (8) were isolated from chloroform fraction of the stem bark. $\beta$-Sitosterol-3-O- $\beta$-D-glucoside (9), 5-Ocaffeoylquinic acid (10) were isolated from the ethyl acetate fraction of the leaves in addition to vanillin (11), benzoic acid (12), protocatechuic acid (13) and flacourtin (14) from the ethyl acetate fraction of the stem bark. The different leaf extracts were subjected to biological study which revealed that $n$-hexane, ethyl acetate and methanol fractions of Flacourtia cataphracta Roxb. are safe to be used as antidiarrheal, anti-inflammatory and antipyretic drug.

\section{INTRODUCTION}

Flacourtia cataphracta Roxb. is a shrub or erect low-branched tree, ${ }^{1 \& 2}$ indigenous to India, ${ }^{1}$ commonly cultivated through out South East Asia ${ }^{1,3 \& 4}$ and is planted in Egypt and many tropical to subtropical parts of the world. It is known in Arabic as Talisfir or Zarnab. ${ }^{1}$ It was reported that the leaves, stem bark and roots have been employed in Indian folk medicine as tonic, diaphoretic, stomachic, in treatment of diarrhea, and also to relieve bronchitis, cough, toothache and other ailments. ${ }^{1-3}$

Some members of the family Flacourtiaceae were reported to contain diversity of natural products as cyanogenic glycosides, ${ }^{5 \& 6}$ triterpenes, ${ }^{7 \& 8}$ sterols, ${ }^{9 \& 10}$ diterpenes, ${ }^{10 \& 11}$ phenolic compounds, ${ }^{7 \& 12}$ benzyl alcohol derivatives ${ }^{7,13-15}$ and few alkaloids. ${ }^{13,16 \& 17}$ Few reports have been found concerning the chemistry of the genus Flacourtia, where flacourtin, ${ }^{18}$ ramoutoside, ${ }^{19} \quad \beta$ sitosterol glucoside, ${ }^{19}$ caffeic acid, ${ }^{20}$ ostruthin, ${ }^{21}$ limonin, ${ }^{21}$ jangomolide ${ }^{21}$ and mortenone ${ }^{22}$ were identified.

No reports could be traced about Flacourtia cataphracta Roxb. cultivated in Egypt, so we decided to carry out phytochemical and biological studies of this plant in order to evaluate the therapeutic effects claimed by traditional medicine and also to provide a new source of natural biologically active compounds. In a previous publication, we reported the macro- and micromorphological characters of the 
leaves, stem and stem bark of the titled plant. $^{23}$

\section{EXPERIMENTAL}

\section{General experimental procedures}

1- Melting points are uncorrected and measured by Koffler hot stage microscope type (ESP, Boctius M).

2- UV spectra were measured in methanol and different ionizing and complexing agents using UVidec-320 spectrophotometer (JAS CO, Japan).

3- Schimadzu infra red-470 spectrophotometer (Japan) was used for measuring IR spectra as $\mathrm{KBr}$ discs.

4- ${ }^{1} \mathrm{H}-\mathrm{NMR}$ and ${ }^{13} \mathrm{C}-\mathrm{NMR}$ were recorded on JEOL TNM-LA 400 and 500 spectrophotometers using TMS as an internal standard.

5- Column chromatography was performed with silica gel 60 (EMerck), Develosil Lop ODS (30$50 \mu$, Nomura Chemicals).

6- Silica gel $60 \mathrm{G} \mathrm{F}_{254}$ and precoated aluminium sheets to silica gel 60 $\mathrm{G}_{254}$ (E-Merck) were used for TLC purposes.

7- The spots were visualized by UV lamp (254, $366 \mathrm{~nm}, \mathrm{VL}, 6 \mathrm{LC}$, Marinc Lavalec-Codex, France) and sprayed with $10 \% \mathrm{H}_{2} \mathrm{SO}_{4}, 5 \%$ $\mathrm{AlCl}_{3}$ or $\mathrm{FeCl}_{3}$.

8- Authentic samples were obtained from Department of Pharmacognosy, Faculty of Pharmacy, Assiut University.

9- Solvent systems:

I- n-Hexane-EtOAc $(9: 1)$.

II- $\mathrm{CHCl}_{3}-\mathrm{MeOH} \quad(9.5: 0.5)$.
III- n-Hexane $-\mathrm{CHCl}_{3}$ - acetic acid $(7.5: 2.5: 0.5)$.

IV- $\mathrm{CHCl}_{3}-\mathrm{MeOH} \quad(9: 1)$.

$\mathrm{V}-\mathrm{CHCl} 3-\mathrm{MeOH} \quad(8: 2)$.

VI- n-Butanol - acetic acid - water

$(4: 1: 2)$.

\section{Plant material}

Leaves and stem bark of Flacourtia cataphracta Roxb. were collected during flowering and fruiting stages in March-June 2001 from the Botanical Garden in Aswan. The plant identity was kindly confirmed by Prof. Dr. Naeem ElKeltawy, Professor of Horticulture, Faculty of Agriculture, Assiut University. A voucher specimen was deposited at the Herbarium of Pharmacognosy Department, Faculty of Pharmacy, Assiut University. The plant material was air-dried, powdered and kept for investigation.

\section{Materials for biological study}

1- Plant extracts for biological screening

i- The air-dried powdered leaves (100 g) was exhaustively extracted by percolation with methanol, the solvent was distilled under reduced pressure leaving $6.5 \mathrm{~g}$ residue, kept for antidiarrheal activity.

ii- The air-dried powdered leaves $(200 \mathrm{~g})$ was successively extracted by percolation with n-hexane, chloroform and ethyl acetate. The solvent in each case was evaporated under reduced pressure giving 4.5, 2.3 and $3.2 \mathrm{~g}$ respectively and kept for toxicological study, anti- 
inflammatory and antipyretic activities.

\section{2- Drugs and chemicals}

Indomethacin (El-Nile Pharm. Co., Egypt), Brewer's dry yeast, castor oil, diphenoxylate (Kahira Pharm. and Chem. Ind. Co., Egypt), Normal saline $0.9 \%$ (El-Nasr Pharmaceutical and Chemical Co., Egypt).

\section{3- Experimental animals}

Adult male albino rats (each 100$120 \mathrm{~g}$ ) were used. The animals were bred and housed under standardized environmental conditions in the preclinical animal house.

\section{Extraction and isolation}

$2 \mathrm{Kg}$ of the air-dried powdered leaves (Lvs) and $1 / 2 \mathrm{Kg}$ stem bark (s.b.) were separately extracted (by maceration) with ethanol $70 \%$ at room temperature for 24 hours four successive times using $5 \mathrm{~L}$ each (Lvs) and 2L each (stem bark) till exhaustion. The total ethanolic extract for each was separately concentrated under reduced pressure to give ( $130 \mathrm{~g}$ Lvs and 30 g s.b) residue, respectively and then separately subjected for successive solvent fractionation with n-hexane, chloroform and ethyl acetate, respectively. Each fraction was separately concentrated under reduced pressure to give the corresponding soluble, n-hexane (40 g Lvs and $8 \mathrm{~g}$ s.b.), chloroform (26 g Lvs and $5 \mathrm{~g}$ s.b.) and ethyl acetate (24 $\mathrm{g}$ Lvs and $12 \mathrm{~g}$ s.b.) fractions, respectively. Each of the obtained fractions was subjected to TLC study followed by chromatographic separation as follows:

1- Column chromatographic fractionation for $20 \mathrm{~g}$ of the $\mathrm{n}$ hexane fraction of the leaves on silica gel $(600 \mathrm{~g}, 150 \times 5 \mathrm{~cm})$ and elution with $n$-hexane and ethyl acetate with increasing polarity, gave three pure compounds (1-3) after rechromtographic and purification trials. TLC of the nhexane fractions of s.b. (syst. I and II, spray with $10 \% \mathrm{H}_{2} \mathrm{SO}_{4}$ ) against the isolated compounds from that of the leaves revealed the presence of compounds $\mathbf{1}, \mathbf{2}$ and $\mathbf{3}$ with the same $R_{f}$ values and colour reactions.

2- Column chromatographic fractionation for $20 \mathrm{~g}$ of the chloroform fraction of the leaves on silica gel $(600 \mathrm{~g}, 150 \times 5 \mathrm{~cm})$ and elution with gradient system of chloroform and methanol, gave five pure compounds (4-8) after rechromatographic and purification trials. TLC of the chloroform fraction of s.b. (syst. I and III spray with $10 \% \mathrm{H}_{2} \mathrm{SO}_{4}$ and $5 \% \mathrm{AlCl}_{3}$ ) against the isolated compounds from that of the leaves, revealed the presence of compounds 4, 6, 7 and 8 with the same $R_{f}$ values and colour reactions.

3- Column chromatographic fractionation for $20 \mathrm{~g}$ of the ethyl acetate fraction of the leaves on silica gel $(600 \mathrm{~g}, 150 \times 5 \mathrm{~cm})$ and elution with gradient system of chloroform and methanol, gave 
only two pure compounds ( 9 and 10) after rechromatographic and purification trials. While column chromatographic fractionation for $12 \mathrm{~g}$ of the ethyl acetate fraction of s.b. using silica gel $(350 \mathrm{~g}$, $120 \times 4 \mathrm{~cm})$ and elution with gradient system of chloroform and methanol afforded four pure compounds (11-14) after rechromatographic and purification trials.

\section{Biological screening}

a) Determination of $\mathrm{LD}_{50}$ of the previously prepared extracts of the leaves according to the reported method, ${ }^{24}$ using male albino rats. $\mathrm{LD}_{50}(\mathrm{~g} / \mathrm{kg})$ for $\mathrm{n}$-hexane and $\mathrm{CHCl}_{3}$ were eight while for EtOAc eleven and for $\mathrm{MeOH}$ ten.

b) Antidiarrheal activity: Adopting castor oil induced diarrhea method, ${ }^{25}$ the activity of the methanolic extract of the leaves was measured in doses of $(100$, 200 and $400 \mathrm{mg} / \mathrm{kg}$ ) suspeneded in $2 \% \mathrm{v} / \mathrm{v}$ aqueous Tween 80 , orally), against diphenoxylate (5 $\mathrm{mg} / \mathrm{kg}$, orally) as standard antidiarrheal drug using five groups (x 6 animal).

c) Antiinflammatory activity: Adopting paw-oedema method, ${ }^{26}$ where oedema was induced by s.c. injection of $20 \% \mathrm{w} / \mathrm{v}$ yeast aqueous suspension in normal saline in the left hind paw under the sub-planter region. The activities of the different extracts of the leaves were measured in doses of $(200$ and $400 \mathrm{mg} / \mathrm{kg}$ suspended in $2.5 \%$ Tween 80 in normal saline, orally) against indomethacin $(8 \mathrm{~g} / \mathrm{kg})$ as standard anti-inflammatory using 10 groups (x 6 animal). The thickness of the paw of the tested animals was measured in $\mathrm{mm}$ using Varinier Caliber after 1, 2, 3 and 4 hours following the administration of the tested extracts.

d) Antipyretic activity: Using Brewer's yeast method, ${ }^{27}$ where pyrexia was induced by s.c. injection of $20 \% \mathrm{w} / \mathrm{v}$ yeast aqueous suspension in normal saline in the left hand paw of the tested rats. The activities of the different extracts of the leaves were measured in doses of $(200$ and $400 \mathrm{mg} / \mathrm{kg}$ suspended in $2.5 \%$ Tween 80 in saline, orally) against indomethacine $(8 \mathrm{mg} / \mathrm{kg}$, orally) as standard antipyretic using 10 groups (x 6 animal). The rectal temperature of each rat was recorded at 1, 2, 3 and 4 hours after administration of the tested extracts.

\section{Compound 1}

White fine needles (ethanol), (30 $\mathrm{mg}$ ), m.p 200-202 ${ }^{\circ}, \mathrm{R}_{\mathrm{f}}=0.43$ (sys. I). IR $v \mathrm{~cm}^{-1}(\mathrm{KBr}): 3450,2940,1380$ and 1037 .

\section{Compound 2}

White needles (acetone), (50 mg), m.p $184-186^{\circ}, \mathrm{R}_{\mathrm{f}}=0.4$ (sys. I). IR $v$ $\mathrm{cm}^{-1}$ (KBr): 3450, 2940, 1385 and 1037. 


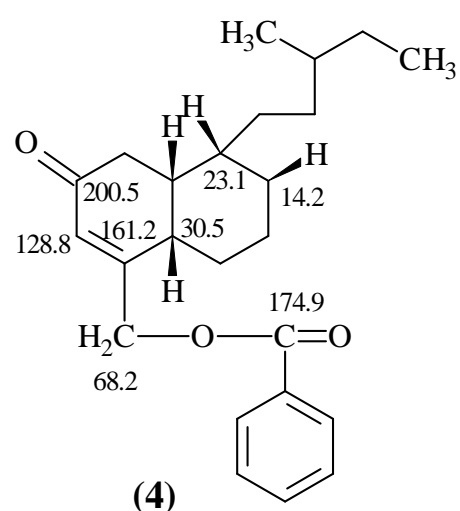

(4)<smiles></smiles>

(6)

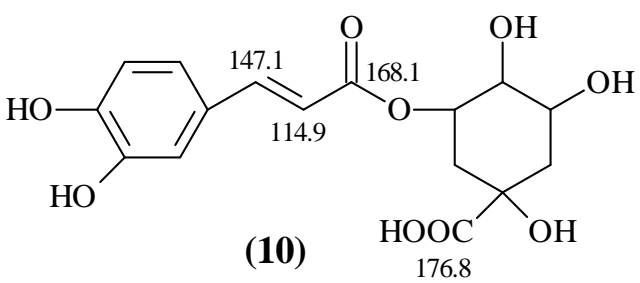

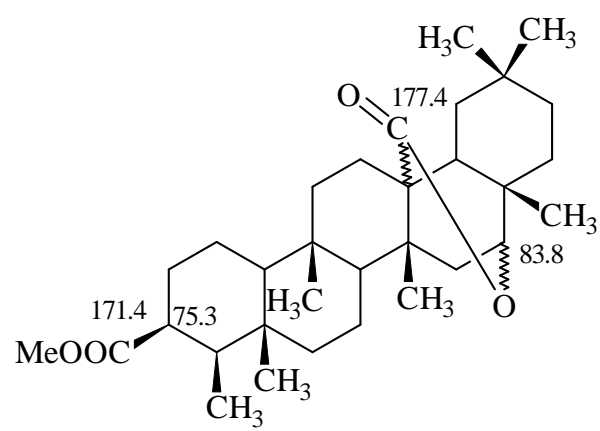

(5)<smiles>[R]c1c(-c2ccc(O)cc2)oc2cc(O)cc(O)c2c1=O</smiles>

$\mathrm{R}=\mathrm{H} \quad$ (7)

$\mathrm{R}=\mathrm{OH}(\mathbf{8})$

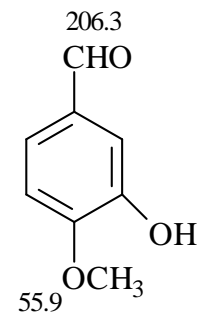

(11)

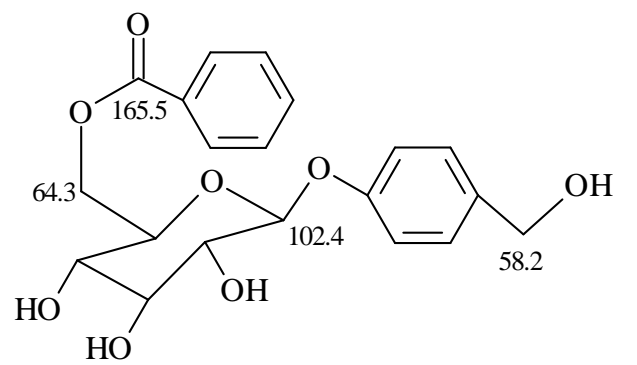

(14) 


\section{Compound 3}

White needles (methanol), (200 $\mathrm{mg}$ ), m.p 134-136 ${ }^{\circ}, \mathrm{R}_{\mathrm{f}}=0.79$ (sys. II).

\section{Compound 4}

Dark yellow oil, $(40 \mathrm{mg}), \mathrm{R}_{\mathrm{f}}=0.71$ (sys. II). IR $v \mathrm{~cm}^{-1}(\mathrm{KBr}): 2945,1765$, 1646, 1520-1420, 1245 and 1056. ${ }^{1} \mathrm{H}-$ NMR $\left(\mathrm{CDCl}_{3}, 500 \mathrm{MHz}\right), \delta: 0.89$ $(3 \mathrm{H}, \mathrm{t}, \mathrm{J}=7.5 \mathrm{~Hz}, \mathrm{Me}-15), 0.90(3 \mathrm{H}$, d, J= $7.5 \mathrm{~Hz}, \mathrm{Me}-16), 0.92(3 \mathrm{H}, \mathrm{d}, \mathrm{J}=$ $7.5 \mathrm{~Hz}, \mathrm{Me}-17), 0.93$ (3H, br.s, Me20), 1.26 (3H, s, Me-19), 1.30-1.69 (m, $\mathrm{CH}_{2}$ groups), $1.89(1 \mathrm{H}$, br.s, $\mathrm{H}-$ 10), $3.16(2 \mathrm{H}, \mathrm{t}, \mathrm{J}=5.7 \mathrm{~Hz}, \mathrm{H}-1), 5.90$ $(1 \mathrm{H}$, br.s, H-3), $6.90(2 \mathrm{H}, \mathrm{ABq}, \mathrm{J}=$ $8.80 \mathrm{~Hz}, \mathrm{H}-18), 7.46(2 \mathrm{H}, \mathrm{t}, \mathrm{J}=7.5$ $\left.\mathrm{Hz}, \mathrm{H}-3^{\prime}, 5^{\prime}\right), 7.52(1 \mathrm{H}, \mathrm{dd}, \mathrm{J}=7.5,1.3$ $\left.\mathrm{Hz}, \mathrm{H}-4^{\prime}\right)$ and $8.08(2 \mathrm{H}, \mathrm{dt}, \mathrm{J}=7.5,1.3$ $\left.\mathrm{Hz}, \mathrm{H}-2^{\prime}, 6^{\prime}\right) .{ }^{13} \mathrm{C}-\mathrm{NMR}\left(\mathrm{CDCl}_{3}, 125\right.$ $\mathrm{MHz}$, $\delta$ : 11.0 (Me-15), 14.2 (Me17), 20.9 (Me-16), 23.1 (Me-20), 23.9 (C-6, C-12), 26.7 (C-14), 28.9 (C-7), 29.7 (C-8, C-11), 30.5 (Me-19), 31.9 (C-31), 36.0 (C-1), 38.9 (C-6, C-9), 47.3 (C-10), 68.2 (C-18), 127.0 (C$\left.3^{\prime}, 5^{\prime}\right), 128.8$ (C-3), 130.2 (C-2',6'), 132.0 (C-1'), 136.0 (C-4'), 161.2 (C4), $174.9(\mathrm{O}-\mathrm{C}=\mathrm{O})$ and $200.5(\mathrm{C}-2)$. EIMS, no molecular ion peak, fragments at $\mathrm{m} / \mathrm{z} ; 325(10 \%), 220$ (2\%), $135(3 \%), 105$ (10\%), 85 (20\%), 77 (31\%) and 43 (39.86\%).

\section{Compound 5}

White plates (methanol), (30 mg), m.p 322-324 ${ }^{\circ}, \mathrm{R}_{\mathrm{f}}=0.87$ (sys. IV). ${ }^{1} \mathrm{H}$ NMR $\left(\mathrm{CDCl}_{3}, 500 \mathrm{MHz}\right), \delta: 0.80$ $(3 \mathrm{H}, \mathrm{d}, \mathrm{J}=7.1 \mathrm{~Hz}, \mathrm{Me}-23), 0.89$ (3H, s, Me-25), 0.90 (3H, s, Me-30), 0.91 (3H, s, Me-24), 0.99 (3H, s, Me-29), 1.19 (3H, s, Me-26), 1.20 (3H, s, Me-
28), 1.5-2 (m, $\left.-\mathrm{CH}_{2}\right), 2.04(3 \mathrm{H}, \mathrm{s}, \mathrm{Me}-$ acetoxy), $3.97(1 \mathrm{H}$, br.t, $\mathrm{H}-16)$ and $4.6(1 \mathrm{H}, \mathrm{dt}, \mathrm{J}=5.7,2.8,2.8 \mathrm{~Hz}, \mathrm{H}-3)$. ${ }^{13} \mathrm{C}-\mathrm{NMR} \quad\left(\mathrm{CDCl}_{3}, 125 \mathrm{MHz}\right) \delta$ : 11.30 (Me-23), 15.80 (Me-24), 16.50 (C-7), 18.20 (Me-25), 18.30 (C-12), 20.70 (Me-26), 21.60 (Me-ester), 21.70 (C-1), 23.70 (Me-28), 28.30 (C-20), 30.41 (C-22), 30.90 (Me-29), 32.70 (C-2, 19), 36.70 (C-17), 36.80 (C-11, 21), 36.90 (Me-30), 37.60 (C9), 39.30 (C-18), 39.4 (C-4, 14), 39.6 (C-15), 41.0 (C-6), 49.90 (C-4), 51.80 (C-13), 57.80 (C-8), 60.0 (C-10), 75.30 (C-3), 83.80 (C-16), 171.40 $(\mathrm{C}=\mathrm{O}$ ester $)$ and $177.40 \quad(\mathrm{C}-27)$. EIMS, no molecular ion peak, fragments at $\mathrm{m} / \mathrm{z}$ (rel. int.): 439 (25\%), 438 (69\%), 423 (75\%), 371 $(18 \%), 370(28 \%), 369(16 \%)$ and $355(11 \%)$.

\section{Compound 6}

Yellow amorphous powder (methanol), (30 mg), $\mathrm{R}_{\mathrm{f}}=0.65$ (sys. IV). UV ( $\left.\lambda_{\max }, \mathrm{nm} \mathrm{MeOH}\right): 225,346$; +NaOMe: 248,322 (sh), 317; $+\mathrm{AlCl}_{3}$ : $225, \quad 346 ;+\mathrm{AlCl}_{3} / \mathrm{HCl}: 225, \quad 346$; +NaOAc: $240 \quad$ (sh), 354 $+\mathrm{NaOAc} / \mathrm{H}_{3} \mathrm{BO}_{3}: 225,245 .{ }^{1} \mathrm{H}-\mathrm{NMR}$ (DMSO-d $6,400 \mathrm{MHz}) \delta: 6.83(4 \mathrm{H}, \mathrm{d}$, $\left.\mathrm{J}=8.5 \mathrm{~Hz}, \mathrm{H}-2^{\prime}, 6^{\prime}, \mathrm{H}-3^{\prime}, 5^{\prime}\right), 6.97(4 \mathrm{H}$, $\mathrm{d}, \mathrm{J}=8.5 \mathrm{~Hz}, \mathrm{H} 2,6, \mathrm{H} 3,5), 7.30(1 \mathrm{H}$, $\mathrm{d}, \mathrm{J}=15.3 \mathrm{~Hz}, \mathrm{H}-\alpha), 7.70(1 \mathrm{H}, \mathrm{d}, \mathrm{J}=$ $15.3, \mathrm{H}-\beta)$ and $10.20(2 \mathrm{H}, \mathrm{s}, \mathrm{OH})$. ${ }^{13} \mathrm{C}-\mathrm{NMR}$ (DMSO-d $\left.6,100 \mathrm{MHz}\right) \delta$ : $112.70 \quad(\mathrm{C}-3,5), \quad 118.20 \quad\left(\mathrm{C}-3^{\prime}, 5^{\prime}\right)$, $122.30(\mathrm{C}-\alpha), 126.80(\mathrm{C}-1), 127.40$ $\left(\mathrm{C}-1^{\prime}\right), 128.60(\mathrm{C}-2,6), 131.50 \quad(\mathrm{C}-$ $\left.2^{\prime}, 6^{\prime}\right), 149.90$ (C- $\left.\beta\right), 153.90$ (C-4), $166.90\left(\mathrm{C}-4^{\prime}\right)$ and $191.30(\mathrm{C}=\mathrm{O})$. EIMS at $\mathrm{m} / \mathrm{z}$ (rel. int.): 240 (0.6\%), 
239 (1.3\%), $185.2 \quad(40 \%), \quad 148.2$ (60\%), $93(100 \%)$ and $43(30 \%)$.

\section{Compound 7}

Yellow amorphous powder (methanol), (20 mg), $\mathrm{R}_{\mathrm{f}}=0.53$ (sys IV). UV ( $\left.\lambda_{\max }, \mathrm{nm} \mathrm{MeOH}\right): 268,338$; +NaOMe: 274, 382; + $\mathrm{AlCl}_{3}: 278$, 354, 384; $+\mathrm{AlCl}_{3} / \mathrm{HCl}: 278,354,384$; +NaOAc: 276, 338; +NaOAc/ $\mathrm{H}_{3} \mathrm{BO}_{3}$ : 268, 338. ${ }^{1} \mathrm{H}-\mathrm{NMR} \quad\left(\mathrm{CD}_{3} \mathrm{OD}, 400\right.$ $\mathrm{MHz}) \delta: 6.21(1 \mathrm{H}, \mathrm{d}, \mathrm{J}=3.16 \mathrm{~Hz}, \mathrm{H}-$ 6), $6.46(1 \mathrm{H}, \mathrm{d}, \mathrm{J}=3.16 \mathrm{~Hz}, \mathrm{H}-8)$ $6.59(1 \mathrm{H}, \mathrm{s}, \mathrm{H}-3), 6.92(2 \mathrm{H}, \mathrm{d}, \mathrm{J}=8.7$ $\left.\mathrm{Hz}, \mathrm{H}-3^{\prime}, 5^{\prime}\right)$ and $7.85(2 \mathrm{H}, \mathrm{d}, \mathrm{J}=8.7$ $\left.\mathrm{Hz}, \mathrm{H}-2^{\prime}, 6^{\prime}\right)$.

\section{Compound 8}

Yellow amorphous powder (methanol), (30 mg), $\mathrm{R}_{\mathrm{f}}=0.50$ (sys. IV). UV ( $\left.\lambda_{\text {max }}, \mathrm{nm}, \mathrm{MeOH}\right): 270,374$; +NaOMe: 288, 442; + $\mathrm{AlCl}_{3}: 276$, 434, 358; + $\mathrm{AlCl}_{3} / \mathrm{HCl}: 274,434,358$; +NaOAc: 276,378 ; +NaOAc/ $\mathrm{H}_{3} \mathrm{BO}_{3}$ : 270, 374. ${ }^{1} \mathrm{H}-\mathrm{NMR}$ (DMSO-d 6 , 500 $\mathrm{MHz})$ \&: 6.17 (1H, br.s., H-6), 6.39 (1H, br.s., H-8), 6.87 (2H, d, J= 8.5 $\left.\mathrm{Hz}, \mathrm{H}-3^{\prime}, 5^{\prime}\right), 7.51(2 \mathrm{H}, \mathrm{d}, \mathrm{J}=8.5 \mathrm{~Hz}$, $\left.\mathrm{H}-2^{\prime}, 6^{\prime}\right)$ and $12.48(1 \mathrm{H}, \mathrm{s}, 5-\mathrm{OH}) .{ }^{13} \mathrm{C}-$ NMR (DMSO-d $6,125 \mathrm{MHz}) \delta: 93.30$ (C-8), 98.20 (C-6), 103.0 (C-10), 115.60 (C-3',5'), 122.0 (C-1'), 129.40 (C-2',6'), 135.7 (C-3), 146.70 (C-2), 156.10 (C-9), 160.0 (C-4'), 160.70 (C-5), 164.0 (C-7) and 175.80 (C-4).

\section{Compound 9}

White granular powder (methanol), (500 mg), $\mathrm{R}_{\mathrm{f}}=0.37$ (sys. IV). ${ }^{1} \mathrm{H}-\mathrm{NMR}\left(\mathrm{C}_{5} \mathrm{D}_{5} \mathrm{~N}, 400 \mathrm{MHz}\right) \delta$ : 0.63 (3H, s, Me-18), 0.83, 0.85 and 0.90 (9H, m, Me-26, 27, 29), 0.89
(3H, s, Me-19), 0.97 (3H, d, J= 5.9 $\mathrm{Hz}, \mathrm{Me}-21), 1.60-2.74$ (m, $\mathrm{CH}_{2}$ \& $\mathrm{CH}$ ), 3.94-4.60 (m, sugar protons), $3.99(1 \mathrm{H}, \mathrm{m}, \mathrm{H}-3 \alpha)$ and $5.33(1 \mathrm{H}$, br.s., H-6). ${ }^{13} \mathrm{C}-\mathrm{NMR}\left(\mathrm{C}_{5} \mathrm{D}_{5} \mathrm{~N}, 400\right.$ MHz) ס: 12.0 (Me-18), 12.1 (Me-29), 19.0 (Me-21), 19.2 (Me-26), 19.4 (Me-19), 20.0 (Me-27), 21.3 (C-11), 23.4 (C-28), 24.5 (C-15), 26.3 (C-23), 28.5 (C-16), 29.4 (C-2), 30.2 (C-25), 32.0 (C-8), 32.2 (C-7), 34.2 (C-22), 36.4 (C-20), 37.5 (C-18), 39.3 (C-4), 36.90 (C-10), 36.92 (C-12), 42.5 (C13), 46.0 (C-24), 50.3 (C-9), 56.2 (C17), 56.8 (C-14), 62.8 (C-6'), 71.6 (C4'), 75.3 (C-2'), 78.4 (C-5'), 78.1 (C3'), 78.6 (C-4), 102.3 (C-1'), 121.9 (C-6) and 140.8 (C-5). Acid hydrolysis: $5 \mathrm{mg}$ of 9 in $5 \mathrm{ml} \mathrm{MeOH}$ and $5 \mathrm{ml} \mathrm{N} / 2$ methanolic sulphuric acid were refluxed for 3 hours, then the aglycone was extracted with $\mathrm{CHCl}_{3}$ and purified and the produced sugar was identified by silica gel PC and system VI.

\section{Compound 10}

Yellowish brown amorphous powder (methanol), (50 mg), $\mathrm{R}_{\mathrm{f}}=0.3$ (sys. V). UV ( $\left.\lambda_{\max }, \mathrm{nm}, \mathrm{MeOH}\right): 330$. ${ }^{1} \mathrm{H}-\mathrm{NMR}$ (DMSO-d 6 , $\left.500 \mathrm{MHz}\right) \delta$ : 1.94-2.16 (4H, m, H-2,6), $3.67(1 \mathrm{H}$, $\mathrm{dd}, \mathrm{J}=8.5,3.1 \mathrm{~Hz}, \mathrm{H}-4), 4.11(1 \mathrm{H}, \mathrm{m}$, H-3), 5.36 (1H, m, H-5), 6.27 (1H, d, $\left.\mathrm{J}=15.8 \mathrm{~Hz}, \mathrm{H}-8^{\prime}\right), 6.76(1 \mathrm{H}, \mathrm{d}, \mathrm{J}=8.2$ Hz, H-5'), 6.94 (1H, dd, J= 8.2, 1.9 Hz, H-6'), 7.03 (1H, d, J= 1.9 Hz, H$\left.2^{\prime}\right)$ and $7.54\left(1 \mathrm{H}, \mathrm{d}, \mathrm{J}=15.8 \mathrm{~Hz}, \mathrm{H}-7^{\prime}\right)$. ${ }^{13} \mathrm{C}-\mathrm{NMR}$ (DMSO-d $\left.6125 \mathrm{MHz}\right) \delta$ : 38.5 (C-2), 39.9 (C-6), 72.3 (C-3), 72.4 (C-5), 74.3 (C-4), 77.7 (C-1), $114.9\left(\mathrm{C}-8^{\prime}\right), 115.6\left(\mathrm{C}-2^{\prime}\right), 116.5(\mathrm{C}-$ 
5'), 123.0 (C-6'), 127.7 (C-1'), 146.8 (C-4'), 147.1 (C-7'), 149.6 (C-3'), $168.1\left(\mathrm{C}^{\prime} \mathbf{9}^{\prime}\right)$ and 176.8 (C-7). EIMS at m/z (rel. int.): $\mathrm{M}^{+} 354.7$ (15\%), 191 (5\%) and $163(18 \%)$.

\section{Compound 11}

Pale yellow amorphous powder with fragrant odour (chloroform), (15 $\mathrm{mg}$ ), $\mathrm{R}_{\mathrm{f}}=0.6$ (sys. II). ${ }^{13} \mathrm{C}-\mathrm{NMR}$ $\left(\mathrm{CDCl}_{3}, 125 \mathrm{MHz}\right)$ \&: $55.9\left(\mathrm{OCH}_{3}\right)$, 114.9 (C-5), 117.9 (C-2), 124.0 (C-6), 130.4 (C-1), 140.9 (C-3), 152.9 (C-4) and 206.3 (CHO).

\section{Compound 12}

White needles (methanol), (40 mg), m.p $122^{\circ}, \mathrm{R}_{\mathrm{f}}=0.5$ (sys. II). ${ }^{1} \mathrm{H}-$ NMR $\left(\mathrm{CDCl}_{3}, 500 \mathrm{MHz}\right) \delta: 7.48(2 \mathrm{H}$, $\mathrm{t}, \mathrm{J}=7.5 \mathrm{~Hz}, \mathrm{H}-3,5), 7.62(1 \mathrm{H}, \mathrm{dt}, \mathrm{J}=$ $7.5,1.5 \mathrm{~Hz}, \mathrm{H}-4)$ and $8.15(2 \mathrm{H}, \mathrm{dd}$, $\mathrm{J}=7.5,1.5 \mathrm{~Hz}, \mathrm{H}-2,6) .{ }^{13} \mathrm{C}-\mathrm{NMR}$ $\left(\mathrm{CDCl}_{3}, 125 \mathrm{MHz}\right), \delta: 128.4$ (C-3,5), 129.3 (C-1), 130.0 (C-2,6), 133.8 (C4) and $172.4(\mathrm{C}=\mathrm{O})$. EIMS m/z (rel. int.) $\mathrm{M}^{+}$at $122(92 \%), 105(100 \%)$, $77(82 \%)$ and $51(50 \%)$.

\section{Compound 13}

Yellowish brown powder (methanol), (30 mg), $\mathrm{R}_{\mathrm{f}}=0.4$ (sys. V). IR $v \mathrm{~cm}^{-1}$ (KBr): $3450,1633,620$ and 473. ${ }^{1} \mathrm{H}-\mathrm{NMR}\left(\mathrm{CD}_{3} \mathrm{OD}, 400 \mathrm{MHz}\right) \delta$ : $6.54(1 \mathrm{H}, \mathrm{dd}, \mathrm{J}=2.9,8.5 \mathrm{~Hz}, \mathrm{H}-6)$, $6.6(1 \mathrm{H}, \mathrm{d}, \mathrm{J}=8.5 \mathrm{~Hz}, \mathrm{H}-5)$ and 6.75 $(1 \mathrm{H}, \mathrm{d}, \mathrm{J}=2.9 \mathrm{~Hz}, \mathrm{H}-2) .{ }^{13} \mathrm{C}-\mathrm{NMR}$ $\left(\mathrm{CD}_{3} \mathrm{OD}, 100 \mathrm{MHz}\right) \delta: 116.5$ (C-5), 117.4 (C-2), 130.3 (C-6), 130.6 (C-1), 149.7 (C-3), 151.9 (C-4) and 170.1 $(\mathrm{C}=\mathrm{O})$.

\section{Compound 14}

White needles (acetone), $(60 \mathrm{mg})$, m.p $212^{\circ}, \mathrm{R}_{\mathrm{f}}=0.3$ (sys. V). UV ( $\lambda_{\max }$, $\mathrm{nm}, \mathrm{MeOH}): 228$ and 285. IR $v \mathrm{~cm}^{-1}$ (KBr): 3475, 2940, 1747, 1717, 1616, 1646, 1385 and 1038. ${ }^{1} \mathrm{H}-\mathrm{NMR}$ $\left(\mathrm{DMSO}_{6}, 400 \mathrm{MHz}\right) \delta$ : $8.98(1 \mathrm{H}$, br.s, phenolic $\mathrm{OH}), 7.96(2 \mathrm{H}, \mathrm{dd}, \mathrm{J}=$ $7.8,2.5 \mathrm{~Hz}, \mathrm{H}-2,6), 7.69(1 \mathrm{H}, \mathrm{t}, \mathrm{J}=$ $7.5, \mathrm{H}-4), 7.55(2 \mathrm{H}, \mathrm{t}, \mathrm{J}=7.5 \mathrm{~Hz}, \mathrm{H}-$ 3,5), $6.88(1 \mathrm{H}, \mathrm{d}, \mathrm{J}=8.7 \mathrm{~Hz}, \mathrm{H}-5 ")$, $6.76(1 \mathrm{H}, \mathrm{s}, \mathrm{H}-2 "), 6.33(1 \mathrm{H}, \mathrm{dd}, \mathrm{J}=$ 8.7, $2.6 \mathrm{~Hz}, \mathrm{H}-6 "), 4.61$ (1H, d, J= 7.0 $\left.\mathrm{Hz}, \mathrm{H}-1^{\prime}\right), 4.54(1 \mathrm{H}, \mathrm{d}, \mathrm{J}=15.1 \mathrm{~Hz}, \mathrm{H}-$ 7"a), 4.36 (1H, d, J= 15.1 Hz, H-7"b), $4.29,4.36,3.26$ (m, sugar protons) and 4.39 ( $1 \mathrm{H}$, br.s., benzoylic $\mathrm{OH})$. ${ }^{13} \mathrm{C}-\mathrm{NMR}$ (DMSO-d $\left.6,100 \mathrm{MHz}\right) \delta$ : 129.7 (C-1), 129.2 (C-2,6), 128.8 (C3,5), 133.2 (C-4), 165.5 (C-7), 64.3 (C-6'), 71.1 (C-4'), 73.4 (C-2'), 73.8 (C-5'), 76.3 (C-3'), 102.4 (C-1'), 147.1 (C-1"), 133.4 (C-2"), 152.7 (C-3"), 116.9 (C-4"), 113.0 (C-5"), 113.8 (C$6 ")$ and 58.2 (C-7"). EIMS m/z (rel. int.), no molecular ion peak, 267 (2.2\%), 140.1 (28.6\%), 122.1 (100\%), $105.1(82 \%)$ and $77.1(40.6 \%)$.

\section{RESULTS AND DISCUSSION}

The ethanolic extracts of the leaves and stem bark were separately concentrated and partionated between n-hexane, $\mathrm{CHCl}_{3}$ and EtOAc. Column chromatography of the n-hexane fraction of the leaves provided compounds 1-3. Compounds 1 and 2 were identified as $\beta$-amyrin and $\alpha$ amyrin by comparison with authentic samples (mmp, IR, co-chromatography), while compound $\mathbf{3}$ was 
proved to be a mixture of $\beta$-sitosterol and stigmasterol (where TLC of acetylated product on a wedged $\mathrm{AgNO}_{3}$ impregnated silica gel (system III), showed two spots with the same $\mathrm{R}_{\mathrm{f}}$ values).

Column fractionation of $\mathrm{CHCl}_{3}$ fraction of the leaves provided compounds 4-8.

${ }^{1} \mathrm{H}-\mathrm{NMR}$ spectrum of compound 4 exhibited typical signals for bicyclic clerodane diterpene skeletone ${ }^{10 \& 28}$ signals at $\delta \quad 3.16$ and 1.89 are suggested for $\mathrm{H}-1$ and $\mathrm{H}-10$ protons in 2-oxo-clerodanes, ${ }^{10 \& 28}$ in addition to five methyl resonances at $\delta 0.89$ 1.26 for two tertiary, two secondary and a primary one. ${ }^{13} \mathrm{C}-\mathrm{NMR}$ spectrum displayed signals at $\delta 14.2$, 23.1 and 30.5 which are characteristic for Me-17, Me-20 and Me-19 respectively of clerodane diterpene. ${ }^{10 \& 28}$ It also revealed a downfield signal at $\delta 200.5$ assigned for $\mathrm{C}$-2 with oxo-substitution ${ }^{29 \& 30}$ which was confirmed by IR band at $1646 \mathrm{~cm}^{-1}$. The presence of benzoyl moiety was observed from ${ }^{1} \mathrm{H}-\mathrm{NMR}$ and MS spectra where the former showed aromatic signals at $\delta 7.46$ $(2 \mathrm{H}, \mathrm{t}, \mathrm{J}=7.5 \mathrm{~Hz}), 7.52(1 \mathrm{H}, \mathrm{dd}, \mathrm{J}=$ $7.5,1.3 \mathrm{~Hz})$ and $8.08(2 \mathrm{H}, \mathrm{dd}, \mathrm{J}=7.5$, $1.3 \mathrm{H}$ ) for monosubstituted benzene ring which was confirmed by four signals at $\delta 130.2,127,132$ and 136 for $\mathrm{C}-2^{\prime}, 6^{\prime}, \mathrm{C}-3^{\prime}, 5^{\prime}, \mathrm{C}-1^{\prime}$ and $\mathrm{C}-4^{\prime}$ in ${ }^{13} \mathrm{C}$-NMR spectrum; in addition to the appearance of mass fragments at $\mathrm{m} / \mathrm{z}$ 105 for $\mathrm{C}_{7} \mathrm{H}_{5} \mathrm{O}$. A mass fragment at $\mathrm{m} / \mathrm{z} 85$ indicated side chain of $\mathrm{C}_{6} \mathrm{H}_{13}$ (fission at C-9/C-11 for clerodane), ${ }^{10,28 \& 31}$ in addition to another fragment at $\mathrm{m} / \mathrm{z} \quad 325$, indicated that the benzoyl moiety could be attached to the bicyclic skeleton not to the side chain. The ester function detected from IR spectrum (band at $v 1765 \mathrm{~cm}^{-1}$ ) was confirmed by a signal at $\delta 174.9$ in ${ }^{13} \mathrm{C}-\mathrm{NMR}$. The benzoyl ester could be attached to $\mathrm{C}-18$, depending upon its chemical shift in ${ }^{1} \mathrm{H}$ - and ${ }^{13} \mathrm{C}-\mathrm{NMR}$ spectra ( $\delta 6.90$ and 68.2 respectively). Comparison of the obtained data with those previously published for a large group of related clerodane derivatives, ${ }^{10,28 \& 30}$ led to the assignment of its structure as 2-oxo18-benozyloxy, 13(16), 14 tetrahydrocleroda-3-ene.

Compound 5 gave positive colour test for triterpene lactones and/or esters. ${ }^{32}$ Its ${ }^{1} \mathrm{H}-\mathrm{NMR}$ spectrum revealed the presence of seven methyl groups at $\delta$ 0.89-1.2 in accordance with those of friedelane skeleton, which are common in Flacourtiaceae. ${ }^{9 \& 33}$ In addition to a signal at $\delta 4.6(1 \mathrm{H}, \mathrm{dt}, \mathrm{J}=5.7,2.8,2.8$ $\mathrm{Hz}$ ) assigned for $\mathrm{H}-3$ oxomethine proton and the small coupling constant between $\mathrm{H}-3, \mathrm{H}-4$ and $\mathrm{H}-2$ protons, indicated $\beta$-configuration of C-3 substituent. ${ }^{34}$ In addition to a broad triplet at $\delta 3.97$ for $\mathrm{H}-16^{34}$ and a signal at $\delta 2.04$ for Me-CO while ${ }^{13} \mathrm{C}-\mathrm{NMR}$ and DEPT experiment showed signals for 32 carbon atoms, representing 8 methyl, 2 carbonyl, 10 methylene, 4 methine, 2 oxo-methine and 6 quaternary carbons. The spectrum also displayed signals attributed to two carbinols at $\delta 75.3$ and 83.8 for $\mathrm{C}-3$ and $\mathrm{C}-16$ and 
carbonyls, an ester one at $\delta 171.4$ and a lactone one at $\delta 177.4$, respectively. The MS data failed to show the molecular ion peak, but showed characteristic fragments at $\mathrm{m} / \mathrm{z} 439$ (M-ester) and other fragments diagnostic for friedo-oleanane triterpenes. ${ }^{34}$ The previously reported data for some lactonized friedooleananes from other flacouriaceous plants revealed that the lactone at $\mathrm{C}$ 27 was joined to $\mathrm{C}-15 \alpha$, but according to our data the lactone is attached to C-16 $\alpha$, consequently 5 could be identified as $3 \beta$-acetoxyD:A friedo-oleanan-27,16 $\alpha$-lactone. ${ }^{34}$

Compound 6 gave positive $\mathrm{FeCl}_{3}$ test, indicating its phenolic nature. Its UV spectral data suggested its chalcone nature; ${ }^{35}$ a bathochromic shift upon addition of $\mathrm{NaOMe}$ and $\mathrm{NaOAc}$ indicated the presence of free 4-OH at ring $\mathrm{B}$ and free 4 '-OH at ring A respectively, while absence of any shift with $\mathrm{AlCl}_{3}$ indicated the absence of 6'-OH. $\quad{ }^{1} \mathrm{H}-\mathrm{NMR}$ spectrum displayed eight aromatic protons, attributed to two units of 1,4disubstituted benzene rings, represented by two doublets with ortho coupling at $\delta 6.83$ and 6.97 respectively. The two proton doublets at $\delta 7.3$ and 7.7 with $\mathrm{J}=15.3 \mathrm{~Hz}$, indicated a trans-configuration of the ethylenic protons, characteristic for $\mathrm{H}-\alpha$ and H- $\beta$ of chalcones. ${ }^{35}$ The signals at $\delta 10.2$ for non hydrogen bonded $\mathrm{OH}$, confirmed the absence of 6'-OH. The presence of one hydroxyl group in each ring was confirmed by EIMS and fragmentation pattern, where it showed $\mathrm{M}^{+}$at $\mathrm{m} / \mathrm{z} 240$ and characteristic fragments at $\mathrm{m} / \mathrm{z} 148$, 120 and 93. The ${ }^{13} \mathrm{C}-\mathrm{NMR}$ spectrum showed eleven signals attributed to fifteen carbon atoms, with a downfield signal at $\delta 191.3$ for $\mathrm{C}=\mathrm{O}$ of chalcone skeleton, in addition to signals at $\delta 122.3$ and 149.9 for $\mathrm{C}-\alpha$ and $\mathrm{C}-\beta$ position. ${ }^{36}$ It could be concluded that $\mathbf{6}$ is $4,4^{\prime}$-dihydroxy chalcone where its ${ }^{1} \mathrm{H}-\mathrm{NMR}$ and MS spectral data are in accordance with those previously published. ${ }^{37}$

Compounds $\mathbf{7}$ and $\mathbf{8}$ gave positive colour reactions for flavonoidal aglycone. ${ }^{38}$ The UV spectral data in methanol for $\mathbf{7}$ indicated its flavone nature while $\mathbf{8}$ was flavonol one. They were identified as apigenin and kaempferol by direct comparison of their spectral data ${ }^{35 \& 38}$ with literature data and co-chromatography with authentic samples.

Column chromatography fractionation of EtOAc fraction of the leaves provided compounds $\mathbf{9}$ and $\mathbf{1 0}$.

Compound 9 gave positive colour reaction for sterols and positive Molish's test indicating its glycosidic nature. The IR spectrum showed broad band at $3450 \mathrm{~cm}^{-1}$ for hydroxyl groups and at $2960 \mathrm{~cm}^{-1}$ for $\mathrm{C}-\mathrm{H}$ stretching. The ${ }^{1} \mathrm{H}-\mathrm{NMR}$ spectrum showed signal at $\delta 5.33$ for $\Delta^{5-6}$. It also showed a multiplet at $\delta 3.99$ attributed to $\mathrm{H}-3 \alpha$. Two tertiary methyl groups (singlets) and four secondary ones are observed while the sugar protons appeared at $\delta$ 4.074.56 and the signal of the anomeric proton was hidden under the solvent signals. ${ }^{13} \mathrm{C}-\mathrm{NMR}$ spectra of compound 9 showed signals for 35 
carbon atoms. Six of them are methyl groups at $\delta$ range from $\delta$ 12.0-20.0 and an olefinic carbons at $\delta 140.8$ and 121.9 , in addition to the characteristic signals for B-glucose carbons. The other ${ }^{13} \mathrm{C}-\mathrm{NMR}$ data were in a good agreement with those published for $\beta$ sitosterol-3-O-D-glucoside. Acid hydrolysis of 9 gave glucose as a sugar moiety and $\beta$-sitosterol as the aglycone which were identified by cochromatography with authentic samples. From the aforementioned data, compound 9 is identified as $\beta$ sitosterol-3-O-D-glucopyranoside by comparison of IR, ${ }^{1} \mathrm{H}$ - and ${ }^{13} \mathrm{C}-\mathrm{NMR}$ spectral data with the literature data, ${ }^{39}$ direct comparison with authentic sample and acid hydrolysis followed by co-TLC for each of the algycone and sugar part with authentic samples.

${ }^{1} \mathrm{H}-\mathrm{NMR}$ spectrum of compound 10 revealed the presence of three aromatic protons characteristic of 1,3,4-trisubstituted benzene ring at $\delta$ $7.03(1 \mathrm{H}, \mathrm{d}, \mathrm{J}=1.9 \mathrm{~Hz}), 6.94(1 \mathrm{H}, \mathrm{dd}$, $\mathrm{J}=8.2,1.9 \mathrm{~Hz})$ and $6.76(1 \mathrm{H}, \mathrm{d}, \mathrm{J}=$ $8.2 \mathrm{~Hz}$ ) for $\mathrm{H}_{-2}{ }^{\prime}, \mathrm{H}-6^{\prime}$ and $\mathrm{H}-5^{\prime}$ respectively, in addition to two doublets at 7.54 and 6.27 with $\mathrm{J}=$ $15.8 \mathrm{~Hz}$ for two trans-olefinic protons, characteristic for caffeoyl derivatives. ${ }^{40}$ Other signals at $\delta 5.39$, 4.11, 3.67 and 1.94-2.16, attributed to quinic acid protons ${ }^{41}$ were also observed. The ${ }^{13} \mathrm{C}-\mathrm{NMR}$ spectral data displayed two characteristic signals for carbonyl carbons at C-9' and C-7 of the acidic moieties at $\delta 168.1$ and 176.8 , respectively, in addition to other characteristic signals. Comparison of the obtained spectral data with those previously published $^{40 \& 41}$ leads to identification of $\mathbf{1 0}$ as 5-O-caffeoylquinic acid (chlorogenic acid).

Column chromatographic fractionation of the EtOAc fraction of s.b, provided four compounds (1114).

Compound 11 gave positive $\mathrm{FeCl}_{3}$ (T.S) test, indicating its phenolic nature, and it has a fragrant odour. Its ${ }^{13} \mathrm{C}-\mathrm{NMR}$ spectrum exhibited six aromatic carbon signals, methoxyl signal at $\delta 55.9$ and a signal at $\delta 206$ indicating the presence of aromatic aldehydic group. $^{42}$ So it was identified as 3-hydroxy-4-methoxy benzaldehyde (vanillin) and confirmed by direct comparison with authentic sample (co-TLC).

${ }^{1} \mathrm{H}-\mathrm{NMR}$ spectrum of compound 12, showed monosubstituted benzene ring pattern and the ${ }^{13} \mathrm{C}$-NMR showed five signals displayed for 7 carbons characteristic for benzoic acid, ${ }^{42}$ which was confirmed by molecular ion peak at $\mathrm{m} / \mathrm{z} 122$ in EIMS and other characteristic fragments at $\mathrm{m} / \mathrm{z}$ 105 and 77. These data leads to identification of $\mathbf{1 2}$ as benzoic acid which was confirmed by co-TLC with authentic.

Compound $\mathbf{1 3}$ gave positive $\mathrm{FeCl}_{3}$ T.S, indicating its phenolic nature. Its ${ }^{1} \mathrm{H}-\mathrm{NMr}$ spectrum revealed the presence of 1,3,4-trisubstituted benzene ring while ${ }^{13} \mathrm{C}-\mathrm{NMR}$ displayed seven carbons, one of them at $\delta \quad 170.1$ characteristic for carboxylic carbonyl group. By comparing the available data was those previously published, $^{43}$ 
compound $\mathbf{1 3}$ was identified as 3,4dihydroxy benzoic acid (protocatechuic acid).

${ }^{1} \mathrm{H}-\mathrm{NMR}$ spectrum of compound 14 revealed the presence of eight aromatic protons, five of them have characteristic pattern for monosubstituted benzene ring (signals at $\delta 7.96,7.69$ and 7.55 respectively) and three other aromatic protons at $\delta 6.88,6.78$ and 6.33 for 1,3,5-trisubstituted benzene. Furthermore, a phenolic and alcoholic protons were observed at $\delta 6.8$ and 4.93 , beside sugar protons and anomeric proton appeared at $\delta 4.7(\mathrm{~d}$, $\mathrm{J}=7.0 \mathrm{~Hz})$ indicating its $\beta$ configuration. $^{43}$ EIMS showed a fragment at $\mathrm{m} / \mathrm{z} 267$ suggesting a benzoylated glucose moiety ${ }^{44}$ with other fragments at $\mathrm{m} / \mathrm{z} 122,105$ and 77. This was confirmed by acid hydrolysis which gave benzoic acid (co-TLC) and glucose (PC, sys. VI). The obtained ${ }^{1} \mathrm{H}-\mathrm{NMR}$ and EIMS data are in accordance with those previously published for the phenolic glucoside ester flacourtin. ${ }^{45}$ The ${ }^{13} \mathrm{C}$ NMR confirmed the structure by appearance of 20 signals, seven of them are at $\delta 129.7,129.2,128.8$, 133.2 and 165.5 (benzoic acid moiety), six for glucose $(\delta 102.4,76.3$ and 64.3) and seven for benzoyl alcohol. From the previous data compound 14 was identified as 3"hydoxy-4"-hydroxy methyl phenyl-6'O-benzoyl- $\beta$-D-glucopyranoside;

flacourtin. To the best of our knowledge, this is the first compilation of the ${ }^{13} \mathrm{C}$-NMR spectra of this compound.
Toxicological study, revealed that the n-hexane (a), $\mathrm{CHCl}_{3}$ (b), EtOAc (c) and methanol (d) extracts of the leaves were safe to be used internally.

The methanol extract (d) of the leaves showed a significant and dose dependent anti-diarrheal activity (Fig. 1) nearly similar to reference drug diphenoxylate $(5 \quad \mathrm{mg} / \mathrm{kg})$. The different extracts $a, b$ and $c$ exhibited a significant anti-inflammatory activity on yeast-induced oedema in a dose dependent way (200 and 400 $\mathrm{mg} / \mathrm{kg}$ ) ranged from intermediate potency in non polar extracts (a and b) to high potency with polar fractions (c and d) compared with indomethacin as a reference compound (Fig 2). While extracts (b and c) showed a well-marked antipyretic activity at different doses (200 and $400 \mathrm{mg} / \mathrm{kg}$ ) for yeast induced fever compared with reference indoemthainc, extracts (a and d) showed no significant effect (Fig. 3).

In conclusion, it is the first report for isolation and identification of such constituents from the studied plant, the first report for isolation of compounds 4, 5, 7,8 and 13 from the genus Flacourtia and the first report for isolation of compounds $6,10,11$ and $\mathbf{1 2}$ from the family.

Since the preliminary phytochemical screening of the tested extractives and isolation of their contents, showed the presence of sterols and/or triterpenes and diversity of phenolic compounds, we can speculate that these constituents might be responsible for the observed 
pharmacological effects, even if other studies are needed, the obtained result seems to support the use of the plant in phytotherapy.

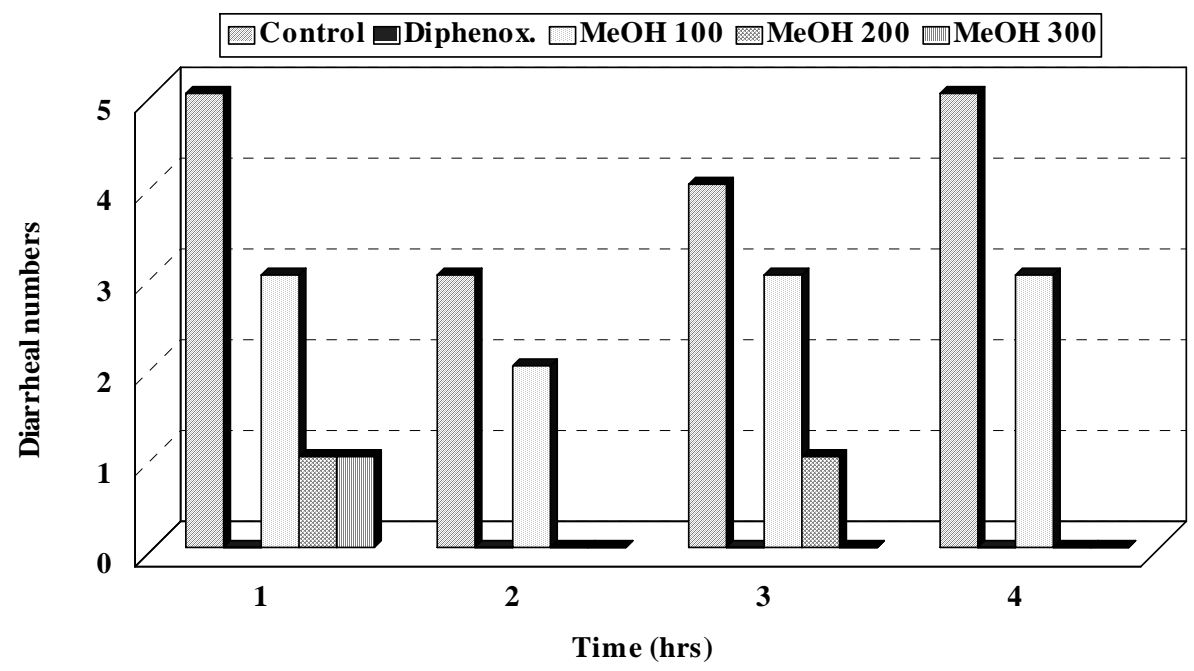

Fig. 1: The anti-diarrheal activity of Flacourtia cataphracta Roxb. leaves.

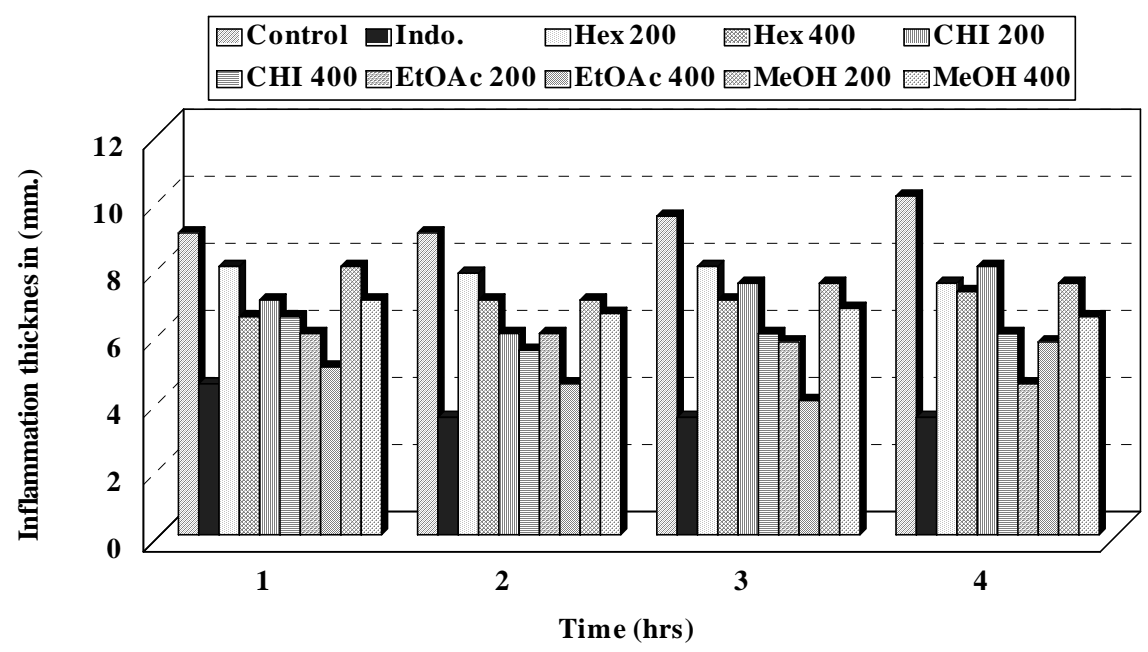

Fig. 2: The anti-inflammatory activity of Flacourtia cataphracta Roxb. leaves. 


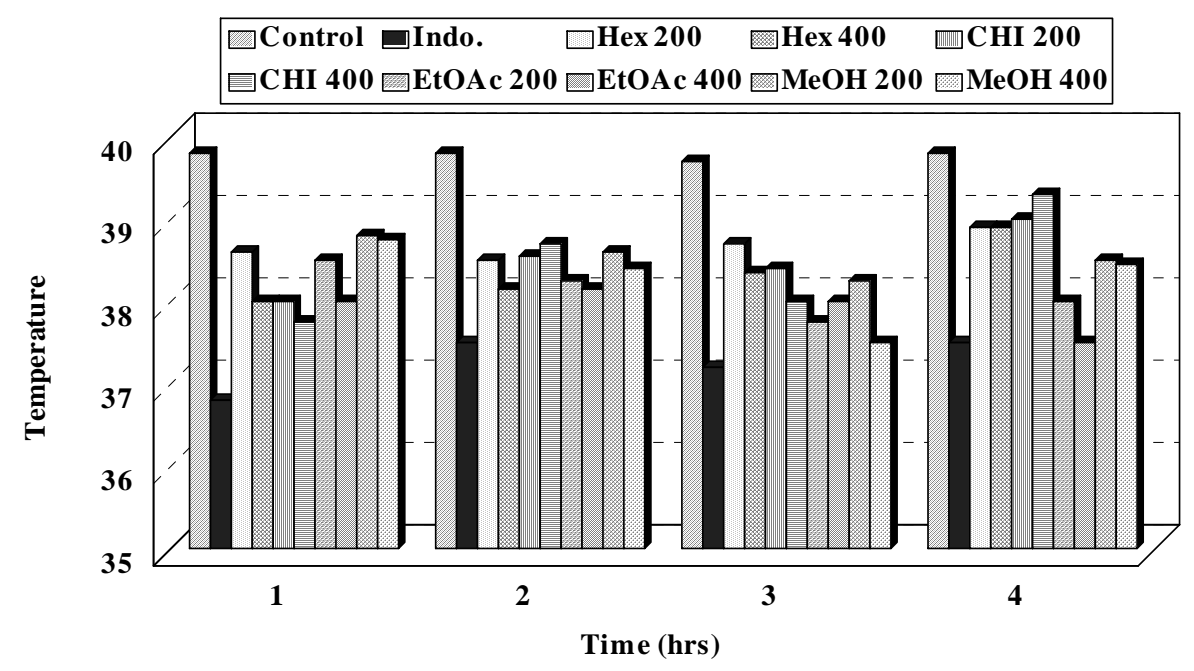

Fig. 3: The anti-pyretic activity of Flacourtia cataphracta Roxb. leaves.

\section{REFERENCES}

1- K. R. Kirtikar, B. D. Basu and L. I. Colonel, "Indian Medicinal Plants", Prakash Publisher, Delhi (1975).

2- L. M. Parry and J. Metzger, "Medicinal Plants of East and South Asia", The MIT Press, Cambridge, Massachuett and London, England (1980).

3- J. Morton, Kei Apple, In: "Fruits of Warm Climates", F. M. Julia, F. L. Miami (1987).

4- J. C. Wills, "A Dictionary of the Flowering Plants and Ferns", Cambridge University Press (1973).

5- J. W. Jaroszewski and E. S. Olafsdottir, Phytochemistry, 26, 3348 (1987).

6- J. W. Jaroszewski, J. V. Andersen and Billeskov, Tetrahedron, 43, 2349 (1987).
7- S. P. Gunasekera, M. U. S. Sultanbawa and S. Balasubramaniam, Phytochemistry, 16, 788 (1977).

8- R. M. Giner-Pons, A. I. Gray, C. Lavaud, G. Massiot, S. Gibbons and P. G. Waterman, ibid., 31, 223 (1992).

9- K. Shaari and P. G. Waterman, ibid., 36, 1021 (1994).

10- M. R. Khan, A. I. Gray and P. G. Waterman, ibid., 29, 2939 (1990).

11- M. R. Khan, A. I. Gray, D. R. Reed, I. H. Sadler and P. G. Waterman, ibid., 29, 1906 (1990).

12- M. C. Reico, R. M. Giner, S. Manerz, G. A. T. Laura, J. Gueho, H. R. Julian, K. Hostettmann and J. L. Rios, Planta Medica, 61, 502 (1995). 
13- L. Crombie, C. F. J. Jones and D. Haigh, Tetrahedron Letters, 27, 5147 (1986).

14- K. Shaari and P. G. Waterman, Phytochemistry, 39, 1415 (1995).

15- S. Gibbons, A. I. Gray, P. G. Waterman, D. C. R. Hockless, B. W. Skelton and A. H. White, J. Nat. Prod., 58, 554 (1995).

16- J. S. Glasby, "Encyclopedia of the Alkaloids", Plenum Press, New York, London (1975).

17- G. E. Trease and W. C. Evans, "Pharmacognosy", Bailliere Tindall, London, $12^{\text {th }}$ Ed. (1996).

18- O. A. Ekabo, N. R. Farnsworth, T. Santisuk and V. Reutrakul, J. Nat. Prod., 56, 699 (1993).

19- V. Satyanarayana, K. G. L. David and G. Srimannarayana, Phytochemistry, 30, 1026 (1991).

20- R. Hegnauer, "Chemotaxonomie der Pflanzen", Birkhauser Verlag, Basel, 1964, p. 4.

21- J. Ahmed, K. Wizarat, K. M. Shamsuddin, A. Zaman and J. D. Connolly, Phytochemistry, 23, 1269 (1984).

22- K. S. Mukherjee, G. Brahmachari and T. K. Manna, J. Indian Chem. Soc., 74, 738 (1997).

23- H. M. Sayed, M. H. Mohammed, F. M. M. Darwish and A. M. Mohamed, Bull. Pharm. Sci., Assiut University, 27, 26 (2004).

24- D. Lorke, Arch Toxicon., 54, 275 (1983).

25- S. C. Mandel, P. K. Mukherjee, K. Saha, M. Pal and B. P. Saha, Nat. Prod. Sci., 3, 100 (1997).
26- R. Domenjoz, W. Thebald and K. Morsodorf, Journal of Arzneim Forsch, 4, 488 (1995).

27- J. M. Teotine, L. P. Faris, A. Gadini and D. Della Bella, Journal of Medicinal Chemistry, 6, 248 (1963).

28- M. R. Khan, A. I. Gray, I. H. Sadler and P. G. Waterman, Phytochemistry, 29, 3591 (1990).

29- S. Gibbons, A. I. Gray and P. G. Waterman, ibid., 43, 635 (1996).

30- S. Gibbons, A. I. Gray and P. G. Waterman, ibid., 41, 565 (1996).

31- J. T. Etse, A. I. Gray, D. W. Thomas and P. G. Waterman, ibid., 28, 2489 (1989).

32- L. F. Fieser and M. Fieser, "Natural Product Related to Phenanthrene", Reinhold Publishing Corp., New York, $3^{\text {rd }}$ Ed. (1949).

33- S. Gibbons, A. I. Gray, D. C. R. Hockless, C. Lavaud, J. M. Nuzillard, G. Massiot, P. G. Waterman and A. H. White, Phytochemistry, 34, 273 (1993).

34- K. Sharri and P. G. Waterman, Phytochemistry, 41, 867 (1996).

35- T. J. Mabry, K. R. Markham and M. B. Thomas, "The Systematic Identification of Flavonoids", Springer, Verlage, New York, Heidelberg, Berlin (1970).

36- P. K. Agrawal, "Carbon-13 NMR of Flavonoids", Elsevier, Amsterdam (1989).

37- O. Hideo, I. Yoshimi, I. Takanori, Y. Kazumasa and Y. Moritami, Phytochemistry, 27, 3993 (1988). 
38- T. A. Giessman, "The Chemistry of Flavonoid Compounds", The MacMillan Co., New York (1969).

39- J. Sakakibara, T. Kaiya, H. Fukuda and T. Ohki, Phytochemistry, 22, 2553 (1983).

40- T. Miyase, M. Ishino, C. Akahori, A. Ueno, Y. Ohkawa and H. Tanizawa, ibid., 30, 2015 (1991).

41- A. Sakushima, S. Hisada, S. Nishibe and H. Branderberger, ibid., 24, 325 (1985).
42- R. M. Silverstein, G. C. Bassler and T. C. Morrill, "Spectrometric Identification of Organic Compounds", John Willey \& Sons, INC, New York, Chichester, Brisbane, Toronto and Singapore, $5^{\text {th }}$ Ed. (1991).

43- I. T. Baldwin and J. C. Schultz, J. C. Science, 221, 227 (1983).

44- P. K. Agrawal, Phytochemistry, 31, 3307 (1992).

45- P. K. Bhaumik, K. P. Guha, G. K. Biswas and B. Mukherjee, ibid., 26, 3090 (1987). 